\title{
Optimization Methods for Minimum Power Bidirectional Topology Construction in Wireless Networks with Sectored Antennas
}

\author{
Arindam K. Das, Robert J. Marks, Mohamed El-Sharkawi, Payman Arabshahi, Andrew Gray
}

\begin{abstract}
We consider the problem of minimum power bidirectional topology optimization in wireless networks with sectored antennas. We first develop a mixed integer linear programming model for optimal solution of the problem with sectored antennas. Subsequently, we discuss a centralized heuristic algorithm which is based on Kruskal's algorithm for the minimum spanning tree problem. However, unlike Kruskal's algorithm which chooses minimum cost edges from a set of edge weights, our algorithm uses an incremental cost mechanism to select edges. This incremental cost mechanism is motivated by the inherently broadcast nature of the wireless medium. We also discuss a simple branch exchange heuristic which can be used to improve the topology generated by the Kruskal-like algorithm. Simulation results indicate that reasonably good solutions can be obtained using the proposed heuristic algorithms.
\end{abstract}

\section{INTRODUCTION}

We consider the problem of minimum transmit power bidirectional topology in multihop wireless networks where individual nodes are typically equipped with limited capacity batteries and therefore have a restricted communication radius. Topology control is one of the most fundamental and critical issues in multihop wireless networks which directly affect the network performance. In wireless networks, topology control essentially involves choosing the right set of transmitter powers to maintain adequate network connectivity. Incorrectly designed topologies can lead to higher end-to-end delays and reduced throughput in error-prone channels. In energyconstrained networks where replacement or periodic maintenance of node batteries is not feasible, the issue is all the more critical since it directly impacts the network lifetime.

In a seminal paper on topology control using transmit power control in wireless networks, Ramanathan and Rosales-Hain [1] approached the problem from an optimization viewpoint and showed that a network topology which minimizes the maximum transmitter power allocated to any node can be constructed in polynomial time. This is a critical criterion in battlefield applications since using higher transmitter power increases the probability of detection by enemy radar. In this paper, we attempt to solve the minimum power topology problem in wireless networks with sectored antennas. Minimizing

A.K. Das and M. El-Sharkawi are with Department of Electrical Engineering, University of Washington, Box 352500, Seattle, WA 98195.

e-mail: \{arindam, melshark\}@ee.washington.edu.

R.J. Marks is with School of Engineering and Computer Science, Baylor University, 1311 S. 5th Street, Waco, TX 76798-7356.

e-mail: Robert_Marks@Baylor.edu.

P. Arabshahi and A. Gray are with Jet Propulsion Laboratory, 4800 Oak Grove Drive, MS 238-343, Pasadena, CA 91109.

e-mail: \{payman, gray\}@ arcadia.jpl.nasa.gov the total transmit power has the effect of limiting the total interference power in the network. It has been shown in [2] that this problem is NP-complete for the special case of omnidirectional (or, single sector) antennas. Related work in the area of minimum power topology construction include [3], [4] and [5], all of which propose distributed algorithms. Specifically, [3] proposes a cone-based distributed algorithm which relies only on angle-of-arrival estimates to establish a power efficient connected topology. Huang et al [4] describe a distributed protocol which is designed for sectored antenna systems. The work in [5] explores the use of relative neighborhood graphs (RNG) for topology control and suggests an algorithm for distributed computation of the RNG. In this paper, we approach the topology design problem from an optimization viewpoint and focus on centralized solution methods. The optimal solution method, in particular, is intended for benchmarking the performance of other heuristics or for offline computation in cases where planned deployment of the network is possible.

We first develop a mixed integer linear programming (MILP) model for optimal solution of the minimum power bidirectional topology problem with sectored antennas. The formulation is flow-based and has a polynomial number of constraints, unlike the model proposed in [6] which uses an exponential number of constraints. Subsequently, we discuss a heuristic algorithm which is based on Kruskal's algorithm [7] for the minimum spanning tree problem. However, unlike Kruskal's algorithm which chooses minimum cost edges from a set of edge weights, our algorithm uses an incremental cost mechanism to select edges. This incremental cost mechanism is motivated by the inherently broadcast nature of the wireless medium. We also discuss a simple branch exchange heuristic which can be used to improve the topology generated using the Kruskal-like algorithm.

The rest of the paper is organized as follows. In Section II, we describe the network model and outline our assumptions. In Section III, we formally define the problem and in Section IV, we develop the MILP model for solving the problem optimally. Section V explains the heuristic algorithms. Finally, simulation results are presented in Section VI.

\section{Network Model}

We consider a static $N$-node network and all nodes are assumed to have $S$-sector antennas. The number of sectors, $S$, is related to the beamwidth, $\theta$ (in degrees), as follows:

$$
S=360 / \theta
$$


Note that $\theta=360(\Rightarrow S=1)$ corresponds to an omnidirectional antenna.

We make several simplifying assumptions on the antenna properties. These are listed below:

- Each sector is assumed to span the angular region $[(s-$ 1) $360 / \theta,(s) 360 / \theta]$ in the 2-D plane, where $1 \leq s \leq S$ is the sector number.

- We ignore sidelobe effects and assume that when sector $s$ is switched on, $100 \%$ of the radiated power is confined within that sector, providing an uniform gain within the angular region spanned by the sector.

- We consider antennas with $100 \%$ efficiency. That is, we ignore any antenna power losses.

Following our simplifying assumptions, the transmitter power at $i$ necessary to support the link $(i \rightarrow j), \mathbf{P}_{i j}$, can be written to be proportional (accounting for link/antenna gains and other factors) to $d_{i j}^{\alpha} / S$, where $d_{i j}$ is the Euclidean distance between nodes $i$ and $j$. If $\left(x_{i}, y_{i}\right)$ are the coordinates of node $i$ and $\alpha$ (typically in the range $2 \leq \alpha \leq 4$ ) is the channel loss exponent, $d_{i j}$ is given by:

$$
d_{i j}=\left[\left(x_{i}-x_{j}\right)^{2}+\left(y_{i}-y_{j}\right)^{2}\right]^{1 / 2}
$$

Without any loss of generality, we set the proportionality constant to be equal to 1 and therefore:

$$
\mathbf{P}_{i j}=d_{i j}^{\alpha} / S
$$

Finally, we address only the transmit power costs in this paper and ignore any reception power costs during the topology setup phase. We plan to address this issues in future, in a broader context of $K$-connectivity, as opposed to simple connectivity assumed in this paper.

\section{Problem Statement}

Let $Y$ be a vector of node transmission powers, the element $Y_{i}$ representing the total transmission power cost of node $i$. For an $S$-sector antenna, $Y_{i}$ can be written as:

$$
Y_{i}=\sum_{s=1}^{S} Y_{i, s}
$$

where $Y_{i, s}$ is the transmission power cost corresponding to sector $s$ of node $i$. We assume that each node has a constraint on the maximum transmitter power it can use per sector, denoted by $Y_{i, s}^{\max }$. That is:

$$
0 \leq Y_{i, s} \leq Y_{i, s}^{\max }: \forall i \in \mathcal{N}, 1 \leq s \leq S
$$

where $\mathcal{N}$ is the set of all nodes in the network and $|\mathcal{N}|=N$.

Also, let $\mathcal{E}$ the set of all bidirected edges ${ }^{1}$. Let the cardinality of the set be $E$; i.e., $E=|\mathcal{E}|$. Using the transmitter power constraint, the set of all bidirectional edges in the network, $\mathcal{E}$, is given by:

$\mathcal{E}=\left\{(i \leftrightarrow j):(i, j) \in \mathcal{N}, i \neq j, \mathbf{P}_{i j} \leq Y_{i, s}^{\max }, \mathbf{P}_{j i} \leq Y_{j, s}^{\max }\right\}$

\footnotetext{
${ }^{1}$ In this paper, the notation $(i \leftrightarrow j$ ) is used to denote a bidirectional link between nodes $i$ and $j$ while a directed link from $i$ to $j$ is represented by ( $i \rightarrow$ $j$ ). The notation $(i, j)$ is used to refer to the node pair.
}

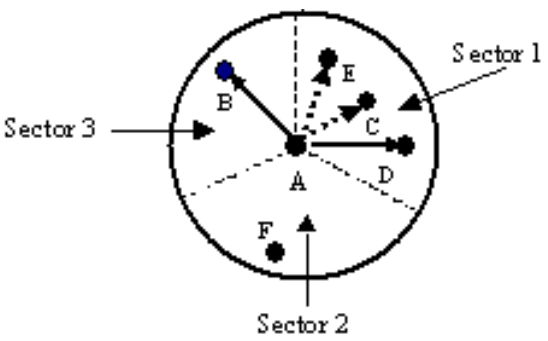

Fig. 1. Illustrating link support with a 3-sector antenna. Nodes $C, D$ and $E$ are located in the same sector, w.r.t $A, D$ being the farthest. Existence of the link $A \rightarrow D$ implies the existence of links $A \rightarrow C$ and $A \rightarrow E$. The total transmission cost of node $A$ is: $Y_{A}=Y_{A, 1}+Y_{A, 3}$, where $Y_{A, 1}=$ $\max \left(\mathbf{P}_{A C}, \mathbf{P}_{A D}, \mathbf{P}_{A E}\right)=\mathbf{P}_{A D}$ and $Y_{A, 3}=\mathbf{P}_{A B}$. Since sector 2 is not used, $Y_{A, 2}=0$.

The third and fourth conditions on the right hand side of (6) enforce the bidirectionality of edges depending on the maximum sector power constraint ${ }^{2}$. For the sake of simplicity, we will, with a slight abuse of notation, also use the set $\mathcal{E}$ to refer to all directed edges, $\{i \rightarrow j\}$, in the graph.

$$
(i \leftrightarrow j) \in \mathcal{E} \Rightarrow(i \rightarrow j) \in \mathcal{E} \text { and }(j \rightarrow i) \in \mathcal{E}
$$

In a wireless network with sectored antennas, the existence of a link from node $i$ to node $j$ also implies the existence of links from $i$ to all nodes which are geometrically closer to $i$ than $j$ and are located in the same sector as $j$, with respect to $i$. For example, in Figure 1, nodes $C, D$ and $E$ are all located in the same sector w.r.t node $A$, node $D$ being the farthest. Existence of the link $A \rightarrow D$ therefore implies the existence of links $A \rightarrow$ $C$ and $A \rightarrow E$. The total transmit power cost of node $A$ is $Y_{A}=$ $Y_{A, 1}+Y_{A, 3}$, where $Y_{A, 1}=\max \left(\mathbf{P}_{A C}, \mathbf{P}_{A D}, \mathbf{P}_{A E}\right)=\mathbf{P}_{A D}$ and $Y_{A, 3}=\mathbf{P}_{A B}$. Since sector 2 is not used, $Y_{A, 2}=0$.

The objective function for the minimum power topological optimization problem is:

$$
\operatorname{minimize}\left(\sum_{i=1}^{N} Y_{i}\right)=\operatorname{minimize}\left(\sum_{i=1}^{N} \sum_{s=1}^{S} Y_{i, s}\right)
$$

Note that instead of minimizing the total transmit power, we could also minimize the per-node maximum transmitter power:

$$
\operatorname{minimize}\left(\max \left\{\sum_{s=1}^{S} Y_{i, s}: 1 \leq i \leq N\right\}\right)
$$

or the per-sector maximum transmitter power:

$$
\operatorname{minimize}\left(\max \left\{Y_{i, s}: 1 \leq i \leq N ; 1 \leq s \leq S\right\}\right)
$$

The objective functions (9) and (10) are equivalent for networks with omnidirectional antennas (i.e., $S=1$ ). It has been shown by Ramanathan and Rosales-Hain [1] that the objective function in (9) can be solved optimally in polynomial time for an omnidirectional antenna system. The algorithm proposed by them is also applicable to the sectored antenna case, if (3) is used to

\footnotetext{
${ }^{2}$ Note that, while the network may have unidirectional edges (e.g., due to uneven transmitter power constraints), we restrict the set of candidate edges to the bidirectional ones only.
} 
compute edge costs. However, it has been shown by Clementi et al [2] that the objective function in (8) is NP-complete for $S=1$. Consequently, it can be inferred that the general $S$ sector optimization problem is NP-complete too.

Finally, we would like to point out that for energy constrained networks, it is desirable that the network topology be optimized taking into account battery residual capacities. This can be accomplished by redefining $\mathbf{P}_{i j}$ as follows:

$$
\mathbf{P}_{i j}=C_{i}^{-\beta}(t)\left(d_{i j}^{\alpha} / S\right)
$$

where $C_{i}(t)$ is the normalized battery residual capacity of node $i$ at time $t\left(0 \leq C_{i}(t) \leq 1\right)$ and $\beta$ is a scaling factor, $\beta \geq 1$.

\section{MILP MODEL}

Let $\left\{F_{i j}: \forall(i \rightarrow j) \in \mathcal{E}\right\}$ be a set of flow variables $\left(F_{i j}\right.$ represents the flow from node $i$ to node $j$ ), with $\mathcal{E}$ defined as in (6). Note that the flow variables correspond to directed edges and therefore $\left|\left\{F_{i j}\right\}\right|=2 E$. The bidirectional topology optimization problem can be interpreted as a single-origin multipledestination uncapacitated flow problem, the number of destinations being equal to $N-1$ (i.e., all nodes other than the source). In flow problems, there is usually an identified source node and a commodity needs to be routed from that source to the destination nodes. In topological optimization problems, however, there's no identified source node. As we will see later, our assumption of bidirectional links allows us to arbitrarily designate a source node without violating the optimality of the solution. Consequently, let us designate node 1 as the source and all other nodes $\{2,3, \cdots N\}$ as the destinations. The corresponding flow problem therefore involves routing $D$ units of supply from node 1 (which has no demand) to all other nodes which have one unit of demand each (and no supply). These supply and demand constraints can be straightforwardly expressed as the following flow conservation equations (see for example [8]):

$$
\begin{gathered}
\sum_{j=2}^{N} F_{1 j}=N-1 ;(1 \rightarrow j) \in \mathcal{E} \\
\sum_{j=2}^{N} F_{j 1}=0 ;(j \rightarrow 1) \in \mathcal{E} \\
\sum_{j=1}^{N} F_{j i}-\sum_{j=1}^{N} F_{i j}=1 ; \forall i \in\{\mathcal{N} \backslash 1\},(i \rightarrow j) \in \mathcal{E}
\end{gathered}
$$

Let us now define a set of indicator variables $\left\{X_{i j}:(i \rightarrow j) \in\right.$ $\mathcal{E}\}$ such that $X_{i j}=1$ if $F_{i j} \geq 1$. The set of constraints which couple the flow variables and the $X_{i j}$ variables is:

$$
(N-1) \cdot X_{i j}-F_{i j} \geq 0 ; \forall(i \rightarrow j) \in \mathcal{E}
$$

For example, given the flow solution in Figure 2(b), the status of the $X_{i j}$ variables are $X_{12}=X_{15}=X_{23}=X_{24}=X_{56}=1$, the rest being equal to 0 . The coefficient of $X_{i j}$ in (15) is due to the fact that the maximum flow out of any node on a single link is equal to $N-1$. Note that the smallest integer value of $X_{i j}$ which satisfies (15) for any nonzero flow out of node $i$ (i.e., if $\left.\sum_{j} X_{i j} \geq 1\right)$ is 1 .

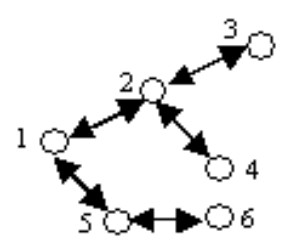

(a)
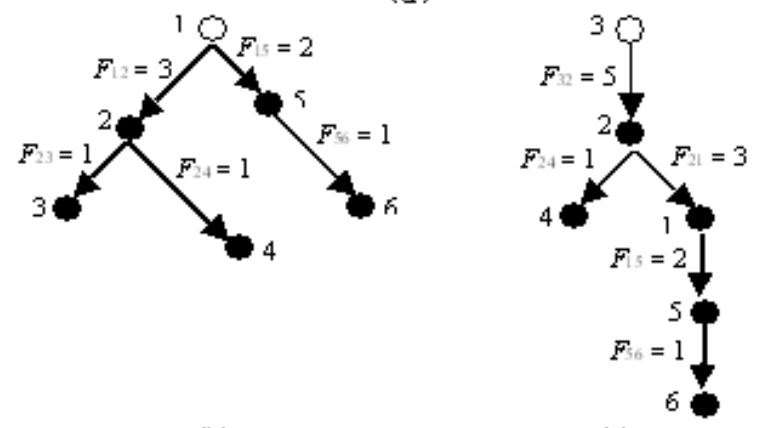

(b)

(c)

Fig. 2. (a) Optimal minimum power topology. (b) Flow solution with node 1 as the source. (c) Flow solution with node 3 as the source.

Finally, we have to relate the indicator variables to the power variables, $\left\{Y_{i, s}\right\}$. To do so, we first define $n e(i, s)$ to be the set of neighbors of node $i$ which are within radio range of $i$ and are located within the same sector, $s$, w.r.t node $i$. For example, in Figure $1, n e(A, 1)=\{C, E, D\}, n e(A, 2)=\{F\}$ and $n e(A, 3)=\{B\}$. Next, we note that the transmit power of both nodes $i$ and $j$ must be adequate in order to support the bidirectional link $\left(i \leftrightarrow j\right.$ ). For example, if $\mathbf{P}_{i j}=\mathbf{P}_{j i}=P$, the transmit powers of both $i$ and $j$ must be equal to $P$ to support the link $(i \leftrightarrow j)$. In other words, the transmit power cost of node $i$ is affected not only by the status of the links outgoing from $i$, but also by the links incident to $i$. Consequently, the power variable $Y_{i, s}$ can be written as:

$$
Y_{i, s}=\max _{j}\left\{X_{i j} \mathbf{P}_{i j}, X_{j i} \mathbf{P}_{j i}: j \in n e(i, s)\right\}
$$

Alternately, bidirectionality can be achieved by imposing constraints on the symmetricity of the indicator variables:

$$
X_{i j}-X_{j i}=0 ; \forall(i \rightarrow j) \in \mathcal{E}
$$

With (16) in place, $Y_{i, s}$ can be expressed as:

$$
Y_{i, s}=\max _{j}\left\{X_{i j} \mathbf{P}_{i j}: j \in n e(i, s)\right\}
$$

or, equivalently,

$$
Y_{i, s}-X_{i j} \mathbf{P}_{i j} \geq 0 ; \forall i, j \in n e(i, s), s=1,2, \cdots S
$$

We now show that defining $Y_{i, s}$ as above makes the optimal solution invariant to the choice of the source node in the flow-balance equations (see eqns. 12, 13 and 14). Consider the optimal topology shown in Figure 2(a). For simplicity, we assume that all nodes have omnidirectional antennas, i.e., $S=1$ in (3). Assume $\mathbf{P}_{15}=\mathbf{P}_{51}=1, \mathbf{P}_{12}=\mathbf{P}_{21}=6$, $\mathbf{P}_{23}=\mathbf{P}_{32}=3, \mathbf{P}_{24}=\mathbf{P}_{42}=4$ and $\mathbf{P}_{56}=\mathbf{P}_{65}=5$. Figures 2(b) and 2(c) show the status of the flow variables 
for different choices of the source node. The $X_{i j}$ variables corresponding to the flow solutions in Figures 2(b) and 2(c) are $X_{12}=X_{15}=X_{23}=X_{24}=X_{56}=1$ and $X_{32}=X_{24}=X_{21}=X_{15}=X_{56}=1$. Note that, despite being a leaf in the flow tree in Figure 2(b), the transmit power cost of node 3 is equal to $\max \left\{X_{23} \mathbf{P}_{23}, X_{32} \mathbf{P}_{32}\right\}=$ $X_{23} \mathbf{P}_{23}=3$, identical to that in Figure 2(c). Similarly, the cost of node 2 in both figures is equal to $\max \left\{X_{23} \mathbf{P}_{23}, X_{24} \mathbf{P}_{24}, X_{21} \mathbf{P}_{21}, X_{32} \mathbf{P}_{32}, X_{42} \mathbf{P}_{42}, X_{12} \mathbf{P}_{12}\right\}$ $=6$. It can easily be verified that the cost of other nodes are the same in Figures 2(b) and 2(c).

The final set of constraints express the integrality of the $X_{i j}$ variables and non-negativity of the $F_{i j}$ and $Y_{i, s}$ variables.

$$
\begin{aligned}
X_{i j} & \geq 0, \text { integer; } \forall(i \rightarrow j) \in \mathcal{E} \\
F_{i j} & \geq 0 ; \forall(i \rightarrow j) \in \mathcal{E} \\
Y_{i, s} & \geq 0 ; \quad \forall i \in \mathcal{N}, s=1,2, \cdots S
\end{aligned}
$$

Note that an upper bound on $Y_{i, s}$ is not required since it is already accounted for in defining the set of valid edges (6). The number of integer variables in the MILP model is equal to $2 E$ while the number of continuous variables is equal to $2 E+S N$. The number of constraints is approximately on the order of $2 E+N(1+2 S)$.

\section{A. Additional constraints}

While the constraints discussed above adequately model the minimum power topology problem, experimental results suggest that the solution time is greatly reduced (by as much as one-fourth) if the model is augmented with the following additional constraints, all of which rely on the symmetric nature of the indicator matrix (16).

- The number of active indicator variables should be equal to $2(N-1)$.

$$
\sum_{i} \sum_{j} X_{i j}=2(N-1) ; \forall(i \rightarrow j) \in \mathcal{E}
$$

- Each node must be connected to at least one other node.

$$
\begin{aligned}
& \sum_{j} X_{i j} \geq 1 ; \forall(i \rightarrow j) \in \mathcal{E} \\
& \sum_{j} X_{j i} \geq 1 ; \forall(j \rightarrow i) \in \mathcal{E}
\end{aligned}
$$

- For each node $i$, there must be at least one outgoing link from its neighbors to the set of all other nodes excluding $i$. This condition is necessary for network connectivity.

$$
\begin{aligned}
& \sum_{j} \sum_{k} X_{j k} \geq 1 ;(j \rightarrow k) \in \mathcal{E}, j \in \cup_{s} n e(i, s), \\
& k \in \mathcal{N} \backslash\left\{i, \cup_{s} n e(i, s)\right\}, \forall i
\end{aligned}
$$

\section{B. Dealing with per-sector maximum power constraint}

Minimizing the total transmit power has the effect of limiting the total interference power in the network. Minimizing the maximum transmit power, on the other hand, is especially critical in military applications since it is directly related to the probability of interception/detection. As mentioned in Section III, the latter criterion can be solved optimally in polynomial time [1]. Unlike a minimum spanning tree, which also
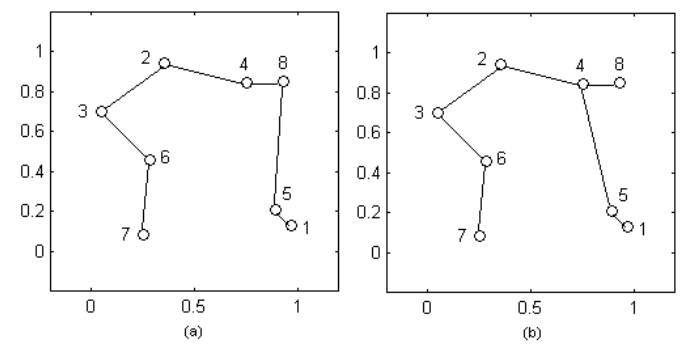

Fig. 3. (a) Optimal topology minimizing the maximum transmit power. The total transmit power is 1.61 and the maximum power is 0.41 , at nodes 5 and 8 . (b) Optimal topology minimizing the total transmit power. The total transmit power is 1.50 and the maximum power is 0.43 , at nodes 4 and 5 .

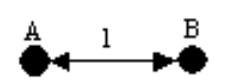

(a)

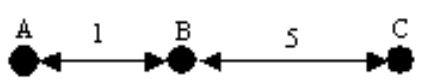

(b)
Fig. 4. Illustrating upper and lower bounds of the MPT, w.r.t the MST. The numbers above the edges represent $\mathbf{P}_{i j}$ 's. (a) Cost of MPT $=2$, which is twice the cost of the MST. (b) Cost of MST $=6$, cost of MPT $=1+\max (1,5)+5=$ 11 , which is equal to the cost of the MST plus the cost of the bottleneck edge in the MST.

minimizes the maximum edge weight, a minimum power topology (MPT) may not minimize the maximum transmit power, as illustrated in Figure 3. Consequently, we may want to solve the MPT problem subject to a constraint on the maximum persector transmit power. Let $\hat{Y}$ be the optimal per-sector maximum transmit power obtained after solving the minimax problem. Redefining the set of valid edges as:

$$
\mathcal{E}=\left\{(i \leftrightarrow j):(i, j) \in \mathcal{N}, i \neq j, \mathbf{P}_{i j} \leq \hat{Y}, \mathbf{P}_{j i} \leq \hat{Y}\right\}
$$

in place of (6) and solving the MILP models will yield a constrained minimum power topology such that the per-sector transmit power of all nodes is not greater than $\hat{Y}$.

\section{Upper and lower bounds on the optimal solution}

Since bidirectionality of a link in a minimum power topology affects the transmit power level of both its end nodes, it is easy to see that its maximum cost is bounded by $2 M S T$, where $M S T$ is the cost of the minimum spanning tree. In other words, one could take the minimum spanning tree, replace each undirected edge by two directed edges and evaluate it on a per-node per-sector basis using (17). Doing so can at most double the cost of the MST, as shown in Figure 4(a) for a trivial 2-node network. On the other hand, since the cost of a node in the MPT problem is the cost of the maximum weight edge incident to it, it is possible to construct a minimum power topology from an MST such that all edges in the MST are accounted for only once in the MPT, except for the bottleneck edge (defined as the maximum cost edge in the MST) which must be accounted for twice. Consider, for example, the MST in Figure 4(b), which has a cost of 6. Evaluated according to the MPT criterion, it can be seen that the power cost of nodes $A, B$ and $C$ are 1, $\max (1,5)$ and 5 respectively. The cost of the $M P T$ is therefore equal to 11 . 

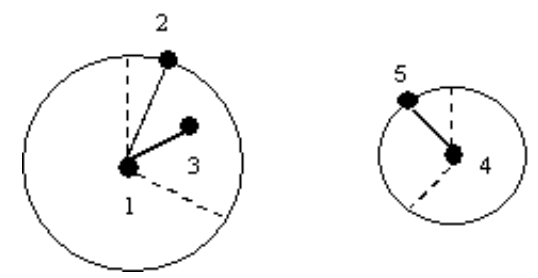

Fig. 5. Illustrating the concept of incremental cost of choosing an edge. Assume that nodes $2,3,4$ and 5 are located in sector 1 w.r.t node 1 and nodes $5,2,3$ and 1 are located in sector 3 w.r.t node 4 . Since node 1 is maintaining bidirected edges with 2 and 3, the transmit power required at its sector 1 antenna is given by: $\mathbf{Y}_{1,1}=\max \left(\mathbf{P}_{12}, \mathbf{P}_{13}\right)=\mathbf{P}_{12}$. Similarly, since node 4 is currently maintaining a bidirected edge with 5 , the transmit power level of its sector 3 antenna is given by: $\mathbf{Y}_{4,3}=\mathbf{P}_{45}$. The incremental cost of choosing the edge $(1 \leftrightarrow 4)$ is defined as the incremental transmit power support required at node 1's sector 1 antenna + the incremental transmit power support required at node 4's sector 3 antenna $=\max \left(0, \mathbf{P}_{1,4}-\mathbf{Y}_{1,1}\right)+\max \left(0, \mathbf{P}_{4,1}-\mathbf{Y}_{4,3}\right)$.

We therefore have the following bounds on the MPT:

$$
(M S T+\text { bottleneck cost in } M S T) \leq M P T \leq 2 M S T
$$

\section{TOPOLOGY CONSTRUCTION AND IMPROVEMENT HEURISTICS}

We first describe a simple heuristic algorithm for constructing the minimum power topology. The algorithm is similar to Kruskal's algorithm for the Minimum Spanning Tree problem, with two salient differences. First, unlike Kruskal's algorithm which minimizes the sum of edge weights, our algorithm attempts to minimize the node weights, the weight of a node being defined as the maximum weight of the edge incident on it. Second, whereas Kruskal's algorithm chooses the minimum weight edge at every iteration from the same set of edge weights, our algorithm implements an incremental cost mechanism to identify the edge to be chosen at any iteration ${ }^{3}$. Before describing the algorithm in more detail, we establish the following notation:

$k=$ iteration number

$\mathbf{Y}^{k}=N \times S$ matrix of node-sector powers after iteration $k$

$\Theta=N \times N$ sector matrix

The $[i, j]$ th element of $\Theta$ specifies the sector in which node $j$ is located w.r.t node $i$.

We illustrate the incremental cost mechanism with an example. In Figure 5, assume that nodes 2, 3, 4 and 5 are located in sector 1 w.r.t node 1 and nodes 5, 2, 3 and 1 are located in sector 3 w.r.t node 4 . Since node 1 is currently maintaining bidirected edges with nodes 2 and 3, the transmit power level of its sector 1 antenna is given by: $\mathbf{Y}_{1,1}=\max \left(\mathbf{P}_{12}, \mathbf{P}_{13}\right)=\mathbf{P}_{12}$. Similarly, since node 4 is currently maintaining a bidirected edge with 5, the transmit power level of its sector 3 antenna is given by: $\mathbf{Y}_{4,3}=\mathbf{P}_{45}$. The incremental cost of choosing the edge $(1 \leftrightarrow 4)$ is defined as the incremental transmit power support required at node 1's sector 1 antenna + the incremental transmit power support required at node 4's sector 3 antenna $=$ $\left(\mathbf{P}_{1,4}-\mathbf{Y}_{1,1}\right)+\left(\mathbf{P}_{4,1}-\mathbf{Y}_{4,3}\right)$. In general, the incremental cost of choosing an edge $(i \leftrightarrow j)$ at iteration $k, I C(i \leftrightarrow j)$, is

\footnotetext{
${ }^{3}$ The incremental cost criterion was proposed by Wieselthier et al [9] in the context of broadcast/multicast routing in wireless networks.
}

defined as the sum of the incremental power supports required at nodes $i$ and $j$, denoted by $I C(i)$ and $I C(j)$.

$$
\begin{aligned}
I C(i \leftrightarrow j) & =I C(i)+I C(j) \\
& =\max \left(0, \mathbf{P}_{i j}-\mathbf{Y}_{i, \Theta_{i j}}^{k-1}\right)+\max \left(0, \mathbf{P}_{j i}-\mathbf{Y}_{j, \Theta_{j i}}^{k-1}\right)
\end{aligned}
$$

For $k=1$, the algorithm chooses the minimum weight edge in $\mathcal{E}$. For $k \geq 2$, the edge which incurs the minimum incremental cost is selected from the set of edges which have not yet been selected, such that it does not form a cycle with the set of edges already selected. Ties, if any, are broken arbitrarily. Suppose that the minimum incremental cost edge at any iteration $k$ is ( $m \leftrightarrow n$ ). The node-sector power matrix is then updated as follows:

$$
\mathbf{Y}_{i s}^{k}:= \begin{cases}\max \left(\mathbf{Y}_{i s}^{k-1}, \mathbf{P}_{m n}\right), & \text { if }(i, s)=\left(m, \Theta_{m n}\right) \\ \max \left(\mathbf{Y}_{i s}^{k-1}, \mathbf{P}_{n m}\right), & \text { if }(i, s)=\left(n, \Theta_{n m}\right) \\ \mathbf{Y}_{i s}^{k-1}, & \text { otherwise }\end{cases}
$$

The algorithm terminates after $N-1$ iterations. A high level description ${ }^{4}$ of the algorithm is provided in Figure 6.

1. Set $k=0$;

2. Initialize the set of feasible edges, $\mathcal{E}$ (see eqns. 6 and 25);

3. Initialize $\mathbf{Y}^{0}=\mathbf{0}$.

4. Initialize the topology: $T=\emptyset$;

5. Increment $k=k+1$;

6. while $(k \leq N-1)$

- Select the edge from $\mathcal{E}$ which incurs the minimum incremental cost (27) and which does not form a cycle with the set of edges chosen previously.

- Suppose that the edge chosen is $(m \leftrightarrow n)$

- $T \leftarrow\{T \cup(m \leftrightarrow n)\} / *$ Add $(m \leftrightarrow n)$ to $T * /$

- $\mathcal{E} \leftarrow \mathcal{E} \backslash(m \leftrightarrow n) ; / *$ Remove edge $(m \leftrightarrow n)$ from $\mathcal{E} * /$

- Update $\mathbf{Y}^{k}$ as in (28).

- Increment $k=k+1$;

end while

5. Cost of the minimum power topology, $T$, is equal to $\sum_{i, s} \mathbf{Y}_{i s}^{t-1}$.

Fig. 6. High level description of an heuristic algorithm for solving the minimum power topology problem with sectored antennas. The algorithm assumes the existence of a spanning tree in the underlying graph.

\section{A. Branch exchange heuristic for topology improvement}

In this section, we describe a simple branch exchange algorithm for improving the initial topology generated using the above Kruskal-like heuristic. Given an initial topology, the algorithm temporarily removes each edge and checks whether the two subtrees created by the edge removal operation can be better reconnected using a replacement edge. If so, the input tree is modified and the branch exchange heuristic is applied on the modified tree. This procedure is repeated until no further improvement is possible. We illustrate the heuristic using an example.

\footnotetext{
${ }^{4}$ Note that Figure 6 does not provide any implementation details. Readers can consult any standard textbook on graph algorithms for an efficient implementation of Kruskal's algorithm.
} 


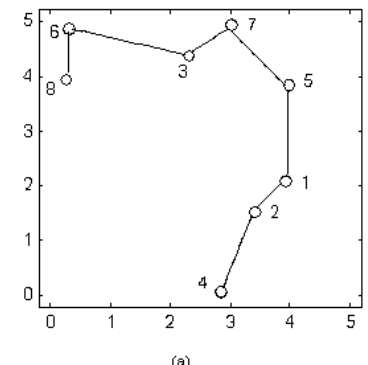

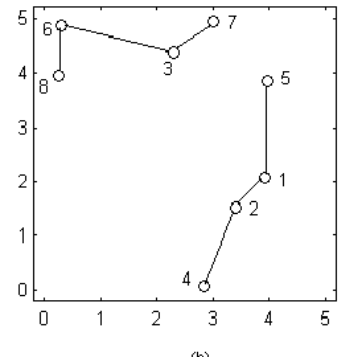

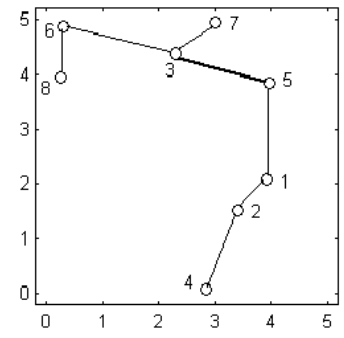

(c)

Fig. 7. (a) Initial topology. The node power vector is shown in (30). The cost of the initial topology is 22.6. (b) Edge (5↔ 7) removed. The two subtrees are:

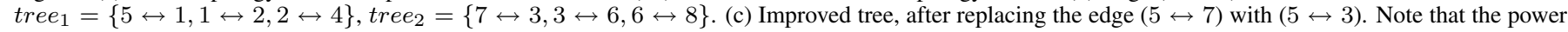
level of node 3 does not change as a result of this replacement operation, since it is still dictated by the branch $(3 \leftrightarrow 6)$. Similarly, the power level of node 5 remains unchanged since it is dictated by the branch $(5 \leftrightarrow 1)$. However, the cost of node 7 drops from 2.2 , required to support the edge $7 \leftrightarrow 5$, to 0.8 , which is necessary to support the edge $(7 \leftrightarrow 3)$. The cost of the improved topology is $21.2,1.4$ units less than the initial topology.

Consider the 8-node network and the initial topology (obtained using the above Kruskal-like heuristic) shown in Figure 7(a). Assume for simplicity that all nodes are provided with omnidirectional antennas.

The power matrix of the network is:

$$
\mathbf{P}=\left[\begin{array}{llllllll}
- & 0.6 & - & - & 3.1 & - & - & - \\
0.6 & - & - & 2.4 & - & - & - & - \\
- & - & - & - & 3.0 & 4.3 & 0.8 & 4.4 \\
- & 2.4 & - & - & - & - & - & - \\
3.1 & - & 3.0 & - & - & - & 2.2 & - \\
- & - & 4.3 & - & - & - & - & 0.8 \\
- & - & 0.8 & - & 2.2 & - & - & - \\
- & - & 4.4 & - & - & 0.8 & - & -
\end{array}\right]
$$

The node-sector power matrix, corresponding to the initial solution is therefore:

$$
\mathbf{Y}=[3.1,2.4,4.3,2.4,3.1,4.3,2.2,0.8]^{\prime}
$$

where ' is the transpose operator. The cost of the initial topology is 22.6 .

Next, suppose we wish to check whether the edge $(5 \leftrightarrow 7)$ can be replaced with a better edge. The two subtrees obtained after its removal is shown in Figure 7(b). In general, let tree $_{1}$ and tree $_{2}$ be the two subtrees created after removing the edge ( $m \leftrightarrow n$ ) from the initial tree, such that $m \in$ tree $_{1}$ and $n \in$ $t_{r e e_{2}}$. The set of potential replacement edges, rep_edges, is then given by:

$$
\begin{gathered}
\text { rep_edges }=\{(i \leftrightarrow j):(i \leftrightarrow j) \neq(m \leftrightarrow n),(i \leftrightarrow j) \in \mathcal{E}, \\
\left.i \in \text { tree }_{1}, j \in \text { tree }_{2}\right\}
\end{gathered}
$$

where $\mathcal{E}$ is the initial edge list, as defined in (6). For our example, the two subtrees are: tree $_{1}=\{5 \leftrightarrow 1,1 \leftrightarrow 2,2 \leftrightarrow 4\}$ and tree $_{2}=\{7 \leftrightarrow 3,3 \leftrightarrow 6,6 \leftrightarrow 8\}$. Using the power matrix (29), it can be easily verified that there is only one potential replacement edge, $(5 \leftrightarrow 3)$, and hence rep_edges $=\{5 \leftrightarrow 3\}$.

To verify whether branch exchange would improve the initial topology, we carry out the following sequence of operations for each edge in the initial tree, denoted by the variable tree:

Step 1: Create a temporary variable, $\mathbf{Y}^{\text {temp }}$, and set it equal to $\mathbf{Y}$, the node-sector power matrix of the initial tree.
Step 2: Remove the edge ( $m \leftrightarrow n$ ) from tree and update $\mathbf{Y}^{\text {temp }}$ as follows:

$$
\begin{aligned}
\mathbf{Y}_{m, \Theta_{m n}}^{t e m p}:=\max \left(\mathbf{P}_{m k}:\right. & k \in\left\{n e\left(m, \Theta_{m n}\right) \backslash n\right\}, \\
& (m \leftrightarrow k) \in \text { tree }) \\
\mathbf{Y}_{n, \Theta_{n m}}^{t e m p}:=\max \left(\mathbf{P}_{n k}:\right. & k \in\left\{n e\left(n, \Theta_{n m}\right) \backslash m\right\}, \\
& (n \leftrightarrow k) \in \text { tree })
\end{aligned}
$$

$\mathbf{Y}_{m, \Theta_{m n}}^{t e m p}$ is set to 0 in (32) if the set $\left\{n e\left(m, \Theta_{m n}\right) \backslash n\right\}$ is empty. Similarly, $\mathbf{Y}_{n, \Theta_{n m}}^{t e m p}$ is set to 0 in (33) if $\left\{n e\left(n, \Theta_{n m}\right) \backslash m\right\}$ is empty. Recall that $n e(i, s)$ is the set of neighbors of node $i$ which are located within the same sector, $s$, w.r.t node $i$.

Step 3: From all edges in the set rep_edges, find the smallest incremental cost edge. As in (27), the incremental cost of adding edge $(i \leftrightarrow j) \in$ rep_edges is given by:

$$
\max \left(0, \mathbf{P}_{i j}-\mathbf{Y}_{i, \Theta_{i j}}^{t e m p}\right)+\max \left(0, \mathbf{P}_{j i}-\mathbf{Y}_{j, \Theta_{j i}}^{t e m p}\right)
$$

Step 4: Let $(i \leftrightarrow j)$ be the smallest incremental cost edge from step-3. The additional cost involved (if any) in adding the edge $(i \leftrightarrow j$ ) is reflected through the following two updates:

$$
\begin{aligned}
\mathbf{Y}_{i, \Theta_{i j}}^{t e m p} & :=\max \left(\mathbf{P}_{i j}, \mathbf{Y}_{i, \Theta_{i j}}^{t e m p}\right) \\
\mathbf{Y}_{j, \Theta_{j i}}^{t e m p} & :=\max \left(\mathbf{P}_{j i}, \mathbf{Y}_{j, \Theta_{j i}}^{t e m p}\right)
\end{aligned}
$$

Step 5: If the sum of the elements of $\mathbf{Y}^{\text {temp }}$ at the end of step 3 is less than the sum of the elements of $\mathbf{Y}$, an improved solution has been found. The initial tree is then modified and the above steps are repeated on the new tree.

$$
\begin{aligned}
\text { tree } & :=\text { tree } \backslash(m \leftrightarrow n) \\
\text { tree } & :=\operatorname{tree} \cup(i \leftrightarrow j) \\
\mathbf{Y} & :=\mathbf{Y}^{\text {temp }}
\end{aligned}
$$

Following the above steps for our example, we have:

Step 1: $\mathbf{Y}^{\text {temp }}=[3.1,2.4,4.3,2.4,3.1,4.3,2.2,0.8]^{\prime}$.

Step 2: Remove the edge $(5 \leftrightarrow 7)$. Given our assumption of omnidirectional antennas, the set $\left\{n e\left(m, \Theta_{m n}\right) \backslash n\right\}$ (see eqn. 32 ), where $m=5$ and $n=7$, is equal to $\{1,7\} \backslash 7=1$. Since the edge $(5 \leftrightarrow 1)$ belongs to the initial tree, we set $\mathbf{Y}_{5,1}^{\text {temp }}:=$ $\max \left(\mathbf{P}_{5,1}\right)=3.1$. Note that the power level of node 5 does not change from step 1 since it is dictated by the cost of the 
higher weight edge incident to it, $(5 \leftrightarrow 1)$. Similarly, the set $\left\{n e\left(n, \Theta_{n m}\right) \backslash n\right\}$ (see eqn. 33) is equal to $\{3,5\} \backslash 5=3$. The corresponding update is: $\mathbf{Y}_{7,1}^{\text {temp }}:=\max \left(\mathbf{P}_{7,3}\right)=0.8$. The node-sector power matrix at the end of this step is:

$$
\mathbf{Y}^{t e m p}=[3.1,2.4,4.3,2.4,3.1,4.3,0.8,0.8]^{T}
$$

Step 3: It can be easily verified that there is only one replacement edge, $(5 \leftrightarrow 3)$.

Step 4: Add the edge $(5 \leftrightarrow 3)$. Using (35) and (36), the updates at this step are: $\mathbf{Y}_{5,1}^{\text {temp }}:=\max \left(\mathbf{P}_{5,3}, \mathbf{Y}_{5,1}^{\text {temp }}\right)=$ $\max (3.0,3.1)=3.1$ and $\mathbf{Y}_{3,1}^{\text {temp }}:=\max \left(\mathbf{P}_{3,5}, \mathbf{Y}_{3,1}^{\text {temp }}\right)=$ $\max (3.0,4.3)=4.3$. The power levels of nodes 5 and 3 do not change from step 2 since they are dictated by the cost of higher weight edges incident to them, $(5 \leftrightarrow 1)$ and $(3 \leftrightarrow 6)$. The node-sector power matrix at the end of this step is therefore:

$$
\mathbf{Y}^{t e m p}=[3.1,2.4,4.3,2.4,3.1,4.3,0.8,0.8]^{T}
$$

The cost of the improved topology is 21.2, 1.4 units less than the initial topology. The improved tree is shown in Figure 7(c). Since an improvement has been found, the improvement algorithm is repeated on the new topology.

We saw in our above example that adding the replacement edge $(5 \leftrightarrow 3)$ at step 3 did not incur any additional cost. Similarly, removing an edge from the initial topology may not reduce the topology cost. Specifically, removing the edge $(i \leftrightarrow j)$ would not reduce the cost if the power levels of the end nodes in the initial topology are greater than the power required to support the link, or, if $\mathbf{Y}_{i \Theta_{i j}}>\mathbf{P}_{i j}$ and $\mathbf{Y}_{j \Theta_{j i}}>\mathbf{P}_{j i}$. Consequently, the edge $(i \leftrightarrow j$ ) need not be considered for removal/replacement during the improvement phase.

\section{Simulation Results}

We have conducted a preliminary study of the performance of the optimal and heuristic methods in 15, 20, 30, 40 and 50-node networks with 3 -sector antennas $(S=3)$. In each case, 100 networks were randomly generated and topology costs were averaged to obtain the mean cost. ' $\alpha$ ' was chosen to be equal to 4 for all cases. The commercially available linear programming solver, LINDO [10], which uses a LP-based branch and brand algorithm to solve MILP problems, was used to compute the optimal solutions. The parameter $Y_{i, s}^{\max }$ was set experimentally such that the average node degree was in the range 4-6 for all $N$. Finally, the normalized residual battery capacity of all nodes was chosen to be equal to 1 , i.e., $C_{i}(t)=1, \forall i$.

Our performance measures for comparing the optimal and heuristic solutions are the mean $\left(P M_{1}\right)$ and the maximum $\left(P M_{2}\right)$ of the percentage relative normalized error or:

$$
100 \times \frac{\sum_{i} Y_{i}(\text { heur })-\sum_{i} Y_{i}(\text { opt })}{\sum_{i} Y_{i}(\text { opt })}
$$

Tables I and II provide a statistical summary of the simulation results, without and with the branch exchange topology improvement algorithm. From Table I, it can be seen that the topology construction algorithm provides reasonably good solutions, within $2.3 \%$ of the optimal in all cases. With the improvement algorithm, the heuristic solutions are within $1.4 \%$ of the optimal. Moreover, the heuristic solutions appear to more closely approximate the optimal with increasing $N$.
TABLE I

Comparison of optimal and heuristic solutions, without improvement.

\begin{tabular}{|c|c|c|}
\hline$N$ & $P M_{1}$ & $P M_{2}$ \\
\hline 15 & 2.29 & 11.26 \\
\hline 20 & 2.04 & 6.09 \\
\hline 30 & 2.01 & 6.01 \\
\hline 40 & 1.47 & 3.21 \\
\hline 50 & 1.23 & 3.19 \\
\hline
\end{tabular}

TABLE II

Comparison of optimal and heuristic solutions, with improvement.

\begin{tabular}{|c|c|c|}
\hline$N$ & $P M_{1}$ & $P M_{2}$ \\
\hline 15 & 1.34 & 10.48 \\
\hline 20 & 0.76 & 5.87 \\
\hline 30 & 0.74 & 4.73 \\
\hline 40 & 0.69 & 2.11 \\
\hline 50 & 0.53 & 2.02 \\
\hline
\end{tabular}

\section{CONCLUSION}

In this paper, we considered the problem of minimum power bidirectional topology optimization in wireless networks with sectored antennas. First, we developed a mixed integer linear programming model for optimal solution of the problem. Next, we discussed heuristic algorithms for topology construction and improvement. The construction heuristic is based on Kruskal's MST algorithm. Simulation results confirm that good results can be obtained using the heuristics, and in very little computation time.

\section{REFERENCES}

1) Ram Ramanathan and Regina Rosales-Hain, "Topology Control of Multihop Wireless Networks using Transmit Power Adjustment", Proc. INFOCOM, 2000.

2) A.E.F. Clementi, P. Penna and R. Silvestri, "Hardness Results for the Power Range Assignment Problem in Packet Radio Networks", Proc. Third International Workshop on Randomization and Approximation in Computer Science (APPROX 1999), Lecture Notes in Computer Science, Vol. 1671, SpringerVerlag, July 1999, pp. 195-208.

3) R. Wattenhofer, L. Li, P. Bahl, Y.M. Wang, "Distributed topology control for power efficient operation in multihop wireless ad hoc networks", Proc. INFOCOM 2001.

4) Zhuochuan Huang, Chien-Chung Shen, C. Srisathapornphat, C. Jaikaeo, "Topology control for ad hoc networks with directional antennas", Proc. ICCN, 14-16 Oct. 2002.

5) S.A. Borbash, E.H. Jennings, "Distributed topology control algorithm for multihop wireless networks, Proc. IJCNN, 12-17 May 2002.

6) E. Althaus, G. Calinescu, I.I. Mandoiu, S. Prasad, N. Tchervenski, A. Zelikovsky, "Power Efficient Range Assignment in Ad-hoc Wireless Networks", Proc. WCNC 2003.

7) J. B. Kruskal. "On the Shortest Spanning Subtree of a Graph and the Travelling Salesman Problem", Proc. of the American Mathematical Society, 1956.

8) Richard Kipp Martin, "Large Scale Linear and Integer Optimization: A Unified Approach”, Kluwer Academic Publishers, Massachusetts, 1999.

9) J.E.Wieselthier, G.D. Nguyen and A. Ephremides, "On the construction of energy-efficient broadcast and multicast trees in wireless networks", Proc. IEEE INFOCOM 2000, pp. 585-594.

10) LINDO solver, www.lindo.com 